\title{
BP lowering beneficial regardless of baseline level?
}

According to Professor Malcolm Law of the Wolfson Institute of Preventative Medicine at the University of London, UK, he and his colleagues have demonstrated that "the terms 'hypertensive' and 'normotensive' should not exist because if there is an optimum blood pressure (BP) level, it is lower than that of most people in western society" and, therefore, "everyone will benefit from lowering BP".

Their meta-analysis included 108 randomized trials of BP-lowering drugs versus placebo or no treatment in patients of mean age 62 years on trial entry, and 46 randomized trials comparing different BP-lowering drugs in patients of mean age 64 years on trial entry. Overall, data were available for 464,000 patients.

All tested BP-lowering drugs were found to decrease the incidence of stroke or events related to coronary heart disease (CHD), regardless of the patient's baseline BP. History of vascular disease

\section{These findings have huge ramifications for the polypill 77}

did not alter these observed effects. Only four trials evaluated the effect of angiotensin-receptor blockers on events related to CHD and no trials studied their effects on stroke. Although thiazides, $\beta$-blockers, angiotensin-convertingenzyme inhibitors, and calcium-channel blockers were all found to decrease the incidence of stroke, calcium-channel blockers seemed to be the most efficacious at doing so. Interestingly, however, calcium-channel blockers were less effective than the other BP-lowering drugs at reducing the incidence of heart failure. In patients who had an infarct within 4 months of enrolment, $\beta$-blockers were most effective in preventing CHD events. In all other patients, the different classes of BP-lowering drugs were found to be similarly effective in the prevention of CHD events. Notably, however, very high doses (more than four times the standard dose) of thiazides were associated with increased risk of sudden death. Compared with one BP-lowering drug at standard dose, three drugs at half their standard doses were about twice as effective in reducing the incidences of $\mathrm{CHD}$ events and stroke.

These findings have huge ramifications for the polypill, a concept first proposed by Wald and Law in 2003. Variations of the polypill are currently being developed and tested by different groups around the world.

Bryony M. Mearns

Original article Law, M. R. et al. Use of blood pressure lowering drugs in the prevention of cardiovascular disease: meta-analysis of 147 randomised trials in the context of expectations from prospective epidemiological studies. BMJ 338, b1665 (2009). 